\title{
Etude du comportement du poulet de chair dans un élevage intensif tropical au Venezuela
}

\author{
P. Richard ${ }^{1}$ M. Vilariño ${ }^{2}$ J.M. Faure ${ }^{3}$ \\ A. León ${ }^{2}$ M. Picard ${ }^{3}$
}

Mots-clés

Poulet de chair - Aviculture Comportement - Adaptation Croissance - Logement de volailles Elevage intensif - Elevage au sol . Climat tropical - Venezuela.

\begin{abstract}
Résumé
Pour identifier des indicateurs comportementaux d'adaptation au milieu, des mesures comportementales par scanning et focal sampling ont été effectuées dans un élevage intensif tropical de 19500 poulets de chair pendant un cycle de production (40 jours). La densité animale autour des mangeoires et abreuvoirs était 2 à 3 fois supérieure à la densité moyenne de l'élevage. La durée d'une séquence d'activité (" couché-couché ») incluant au moins un picorage de l'aliment, de l'eau, de la litière ou une toilette était en moyenne de $133,5 \pm 9,5$ s à 10 jours et diminuait régulièrement pour atteindre $50,0 \pm 1,6 \mathrm{~s}$ à 40 jours. Avec l'âge, les séquences devenaient presque toutes dédiées à une seule activité (mono-comportementales, essentiellement manger ou boire), le comportement d'exploration par picorage de la litière quant à lui régressait. Au cours d'une journée, la durée d'une séquence d'activité diminuait pendant les périodes les plus chaudes et augmentait le soir, mais le poulet, lorsqu'il était actif, présentait le même éthogramme quelle que fût l'heure. En cas de problème d'élevage les comportements d'exploration de la litière augmentaient à nouveau, ce qui pouvait constituer un signal précoce. Les mesures de la densité de répartition des animaux, de la durée d'une séquence d'activité et de la fréquence du picorage de la litière ont un intérêt pratique à condition d'être très précisément définies et réalisées dans des conditions calmes.
\end{abstract}

L'aviculture est un secteur de l'élevage en réelle croissance dans les pays tropicaux. Les productions avicoles représentent 40 p. 100 des protéines d'origine animale consommées par une population vénézuélienne essentiellement urbaine. L'élevage rationnel de souches spécialisées a connu une expansion rapide. Les productions avicoles ont quadruplé depuis les années 70. Cependant, comme dans la plupart des pays tropicaux, la dépendance économique, induite par l'importation d'une grande proportion des matières premières alimentaires, impose une efficacité de transformation élevée à cette filière (10).

1. CIRAD-EMVT, Campus international de Baillarguet, BP 5035, 34032 Montpellier Cedex 1, France

2. Instituto de Investigaciones Zootécnicas, Fondo Nacional de Investigaciones Agropecuarias, Apdo. Postal 4653, Maracay 2101, Venezuela

3. Station de recherches avicoles, INRA, 37380 Nouzilly, France
La distribution ad libitum d'un aliment complet équilibré a longtemps fait sous-estimer l'importance de l'observation du comportement alimentaire des animaux. Les recherches sur l'alimentation des volailles sont basées sur l'étude de la composition du régime, de sa valeur nutritionnelle et de sa rentabilité économique globale, en tenant peu compte des comportements individuels des volailles. L'éthologie a développé depuis de nombreuses années des méthodes précises pour étudier le comportement de différentes espèces animales. La convergence de ces deux types de recherche peut permettre de mieux comprendre comment l'animal évolue et s'adapte à son environnement en utilisant pour cela l'expression de ses comportements (6).

Les observations faites sur des volailles en élevage intensif montrent qu'elles effectuent des accès alimentaires courts et fréquents (7) et que le temps consacré à la consommation d'aliment varie beaucoup en fonction des autres activités possibles (3). Le « milieu de vie » de l'animal dans les élevages intensifs se résume à la présence d'une litière, de congénères, de l'eau et d'un aliment «bien » 
équilibré disponible en permanence à quelques centimètres de son bec. Dans un tel environnement, l'accès à l'aliment nécessite moins d'effort qu'en milieu ouvert et peut représenter une « occupation sociale » (11). Les volailles dans leurs stratégies d'ingestion alimentaire, maximisent le rapport profit/coût (effort) défini comme la quantité d'énergie ingérée par unité d'effort consacré à la prise de l'aliment (4).

Dans un poulailler tropical, de nombreux facteurs environnementaux (lumière, température, hygrométrie, pratiques d'élevage...) diffèrent des conditions de l'élevage avicole dans les pays tempérés. Les techniques d'observation du comportement des volailles ont surtout été pratiquées en station de recherche sur un nombre restreint d'animaux (19). Par contre peu d'études ont eu lieu dans des conditions réelles de production $(7,8,12,13)$. L'objectif du travail présenté ici a été de mesurer les activités des poulets de chair dans un élevage tropical intensif au Venezuela, en utilisant plusieurs techniques d'observation, pour analyser l'intérêt et les limites de leur applicabilité.

\section{MATERIEL ET METHODES}

Les observations se sont déroulées au cours de la saison des pluies, de mai à juillet, dans un élevage industriel de poulets de chair intégré au groupe Protinal (Valencia, Venezuela). Les observations étaient réparties en cinq phases de quatre jours pendant l'ensemble de la période de croissance d'un lot de poulets (3-5, 9-12, 19-22, 30-32 et 37-40 jours d'âge, tableau I). La température au cours de la période expérimentale était en moyenne de $24,7 \pm 2,8^{\circ} \mathrm{C}$ pour les minima et $32,1 \pm 2,7^{\circ} \mathrm{C}$ pour les maxima, l'hygrométrie était en moyenne de $59 \pm 11$ p. 100 pour les minima et $88 \pm 8$ p. 100 pour les maxima.
Le poulailler d'élevage au sol était un bâtiment à structure ouverte orienté selon un axe est-ouest. Les parois latérales étaient constituées d'un grillage reposant sur un muret d'une hauteur d'environ $20 \mathrm{~cm}$ en moyenne ce qui permettait une admission basse d'air au niveau des volailles (figure 1). La ventilation, de type statique (en fonction des vents dominants) au cours des 4 premières phases, devenait dynamique (cinq ventilateurs) au cours de la dernière phase. La toiture constituée de tôles d'aluminium était largement débordante de manière à protéger les animaux de la pluie et du soleil. A partir du $31^{\mathrm{e}}$ jour d'élevage un système d'arrosage du toit fonctionnait pendant les heures les plus chaudes de la journée. La litière était composée de balles de riz (hauteur $=10 \mathrm{~cm}$ en moyenne). Le bâtiment d'une surface totale de $2160 \mathrm{~m}^{2}$ (180 m x $12 \mathrm{~m}$ ) était compartimenté au cours de la période d'élevage, la surface disponible augmentant progressivement avec l'âge des poulets (tableau I). Les mangeoires à remplissage manuel (type «trémie », $\varnothing: 40 \mathrm{~cm}$ ) et les abreuvoirs automatiques (type « cloche », $\varnothing: 35 \mathrm{~cm}$ ) étaient répartis de manière linéaire selon un ordre régulier (figure 1). Pendant les 7 premiers jours d'élevage, des plateaux d'alimentation $(\varnothing: 40 \mathrm{~cm})$ étaient ajoutés et $16 \mathrm{ra}-$ diants à gaz (1,20 $\mathrm{m}$ au-dessus du sol) assuraient une régulation de la température ambiante entre 30 et $35^{\circ} \mathrm{C}$. Des rideaux en plastique fermaient le bâtiment pendant la première semaine d'élevage et étaient ensuite enlevés progressivement en fonction des conditions climatiques. Les 19500 poussins de chair de 1 jour, non sexés, de souche Cobb avaient un poids moyen de $40 \mathrm{~g}$ le jour de la livraison.

L'alimentation était distribuée sous forme de farine du $1^{\mathrm{er}}$ au $19^{\mathrm{e}}$ jour et sous forme de granulés friables à partir du $20^{\mathrm{e}}$ jour. L'éclairement était constant $24 \mathrm{~h}$ sur 24 (lumière naturelle diurne + lumière artificielle nocturne) tout au long de la période d'élevage. La température et l'hygrométrie ambiantes étaient relevées lors de chaque période d'observation; les températures minima et maxima étaient lues chaque matin à $8 \mathrm{~h}$.

\section{Tableau I}

Caractéristiques de la conduite d'élevage au cours des différentes phases d'observation

\begin{tabular}{|c|c|c|c|c|c|}
\hline & Phase 1 & Phase 2 & Phase 3 & Phase 4 & Phase 5 \\
\hline Age des poulets (en jours) & 3 à 5 & 9 à 12 & 19 à 22 & 30 à 32 & 37 à 40 \\
\hline Température $\left({ }^{\circ} \mathrm{C}\right)(1)$ & $31,8 \pm 1,7$ & $30,2 \pm 2,5$ & $30,5 \pm 2,1$ & $29,4 \pm 2,1$ & $25,8 \pm 1,9$ \\
\hline Hygrométrie (\%) (1) & $65 \pm 5$ & $63 \pm 13$ & $65 \pm 9$ & $70 \pm 9$ & $79 \pm 6$ \\
\hline Surface de l'élevage $\left(\mathrm{m}^{2}\right)$ & 540 & 720 & 1404 & 2160 & 2160 \\
\hline Densité (poulets/m²) & 36 & 27 & 14 & 9 & 9 \\
\hline Nombre de mangeoires & 146 & 223 & 438 & 680 & 680 \\
\hline Surface de la mangeoire $\left(\mathrm{m}^{2}\right)(2)$ & 0,20 & 0,28 & 0,38 & 0,50 & 0,50 \\
\hline Nombre d'abreuvoirs & 71 & 71 & 140 & 212 & 212 \\
\hline Surface de l'abreuvoir $\left(\mathrm{m}^{2}\right)(2)$ & 0,16 & 0,24 & 0,33 & 0,44 & 0,44 \\
\hline Nombre de plateaux & 49 & - & - & - & - \\
\hline Surface du plateau $\left(\mathrm{m}^{2}\right)(2)$ & 0,20 & - & - & - & - \\
\hline Nombre de radiants & 16 & - & - & - & - \\
\hline Nombre d'ampoules (3) & 6 & 8 & 16 & 25 & 25 \\
\hline
\end{tabular}

(1) Moyennes et écarts-types aux heures d'observation.

(2) Surface correspondant à la somme de la surface théorique occupée par une mangeoire (ou un abreuvoir) plus l'espace occupé par un poulet « proche » (à moins d'un poulet de distance) autour de cette mangeoire (ou abreuvoir).

(3) Puissance d'une ampoule : 160 watts. 

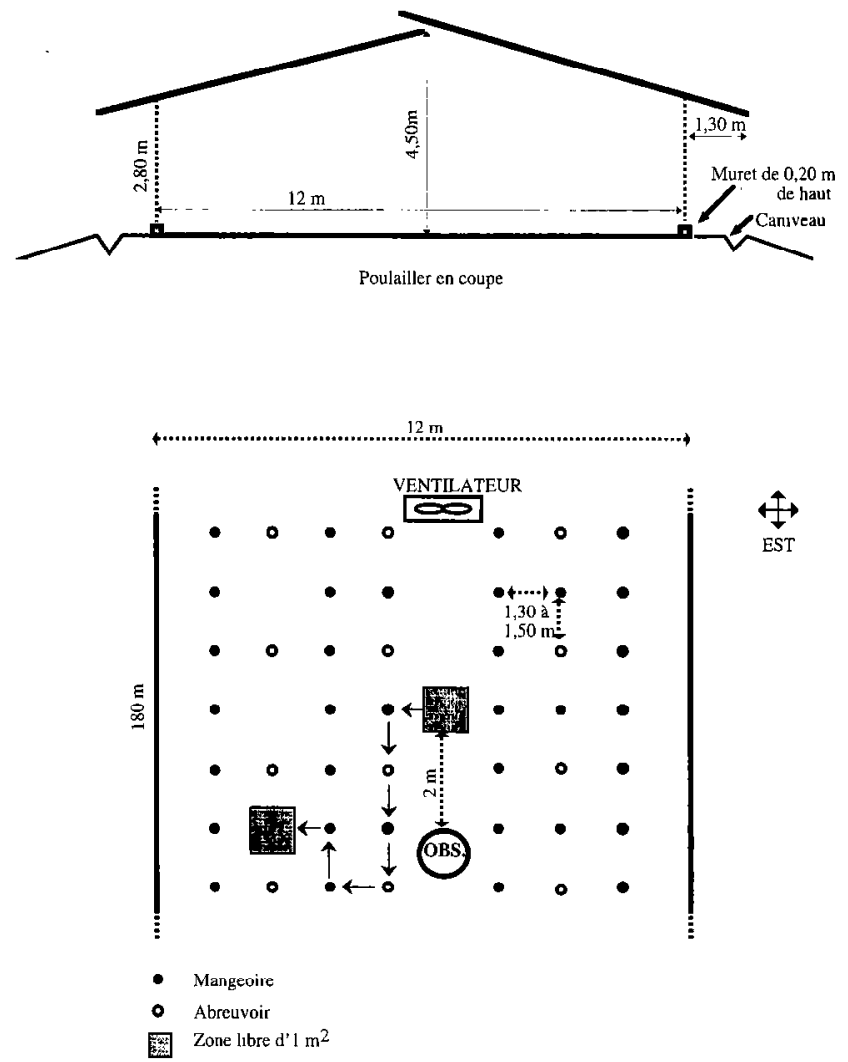

Figure 1 : représentation d'une section de poulailler et des zones observées par scanning.

Les observations comportementales étaient effectuées au cours de cinq phases de quatre jours consécutifs, chaque phase correspondant à une tranche d'âge du poulet (tableau I).

Deux méthodes d'observation étaient utilisées : l'échantillonnage instantané répété ou scunning et l'échantillonnage focalisé sur un individu ou focal sampling (5).

Le scanning consistait en un comptage, à intervalles réguliers chronométrés, du nombre de poulets ayant une même activité sur un site dunné précisément délimité el identifié (mangeoire, abreuvoir, zone libre marquée par des pierres). Un scan représentait le comptage successif de 8 sites d'observation (figure 1), soit 4 mangeoires, 2 abreuvoirs et 2 zones d'une surface d'environ $1 \mathrm{~m}^{2}$ ne contenant ni mangeoire ni abreuvoir. A chaque scan, le nombre de poulets était enregistré dans les situations suivantes:

- les poulets proches (à moins d'un poulet de distance, taille variable en fonction de l'âge) d'une mangeoire (ou d'un abreuvoir) en train de manger (ou de boire) ;

- les poulets proches des équipements mais qui ne mangeaient pas (ou ne buvaient pas) ;

- les poulets debout et les poulets couchés au niveau des zones libres.

La périodicité des scans était fixée à 2 min, soit 30 scans successifs par heure.

Le focal sampling est l'enregistrement continu de l'activité d'un poulet, pris au hasard, à partir de l'instant où il se lève jusquà à l'instant où il se recouche ; il s'agit donc d'une séquence dite «couché-couché ». Les observations de focal sampling étaient en- registrées directement sur un micro-ordinateur portable (AST) utilisant le logicicl d'obscrvation « The Observer 3.0 » (Noldus, Netherlands). Ce logiciel a permis de redéfinir les touches du clavier de l'ordinateur en attribuant à chacune un comportement, ce qui permettait d'enregistrer en temps réel les différentes activités des animaux. A partir de chaque fichier séquentiel la durée, la fréquence, la latence et les enchaînements des différentes activités du poulet étaient calculés.

Les variables comportementales enregistrées ont pris en compte trois types d'information :

- la proximité du poulet par rapport à la mangeoire : proche =' à moins d'un poulet de distance, loin $=$ à plus d'un poulet de distance (au départ dans la zone « loin », les poulets observés étaient choisis au hasard) ;

- l'activité du poulet : cinq comportements exclusifs ont été définis :

«Mange » : le poulet était en face de la mangeoire - action de manger ;

« Boit » : le poulet était en face de l'abreuvoir - action de boire ;

«Toilette» : le poulet se nettoyait les plumes ;

«Explore la litière » : le poulet grattait ou picorait la litic̀rc ;

« Debout »: le poulet était debout, immobile, ou se déplaçait, mais ne pratiquait aucune des activités décrites précédemment ;

- le picorage (comportement non exclusif) comptabilisait le nombre de coups de bec donnés à l'aliment, à l'eau, à la litière ou aux congénères.

De plus, pour chaque fichier, le jour d'observation, la période de la journée (heures d'observation), la température et l'hygrométrie, ainsi que diverses remarques concernant le déroulement de l'enregistrement (météo, interventions diverses du personnel, animaux extérieurs...) étaient enregistrés. L'ob'servateur se plaçait dans l'axe médian du poulailler (figure 1), assis sur une chaise surélevéc (figure 2). Les observations commençaient chaque fois après une période de 5-10 min d'adaptation des poulets à la présence de l'observateur.

Les contrôles se déroulaient pour chaque phase selon des horaires répartis au cours du nycthémère et permettant d'alterner les deux méthodes d'observation, soit de la manière suivante :

- $1^{\text {er jour : }}$

focal sampling de 16 à $18 \mathrm{~h}$ et de 20 à $22 \mathrm{~h}$;

$-2^{\mathrm{e}}$ et $3^{\mathrm{e}}$ jour :

scanning de $9 \mathrm{~h} 30$ à $10 \mathrm{~h} 30$ et de 15 h 30 à 16 h 30 ;

focal sampling de 8 à 9 h, de 11 à 12 h, de 14 à 15 h et de 17 à $18 \mathrm{~h}$;

$-4^{\mathrm{e}}$ jour :

focal sampling de 4 à 6 h et de 10 à $12 \mathrm{~h}$.

Les scannings étaient d'abord relevés sur papier dans le poulailler puis enregistrés sur le tableur Excel. Le nombre de poulets " actifs ", c'est-à-dire proches des équipements et en train de manger ou de boire, était dissocié du nombre de poulets « passifs », c'est-à-dire proches des équipements mais ayant une activité autre que celle de manger ou de boire. Le nombre total de poulets actifs ou passifs était ensuite calculé par type de site, par jour et par période (matin/après-midi) au cours d'une heure de scanning. 


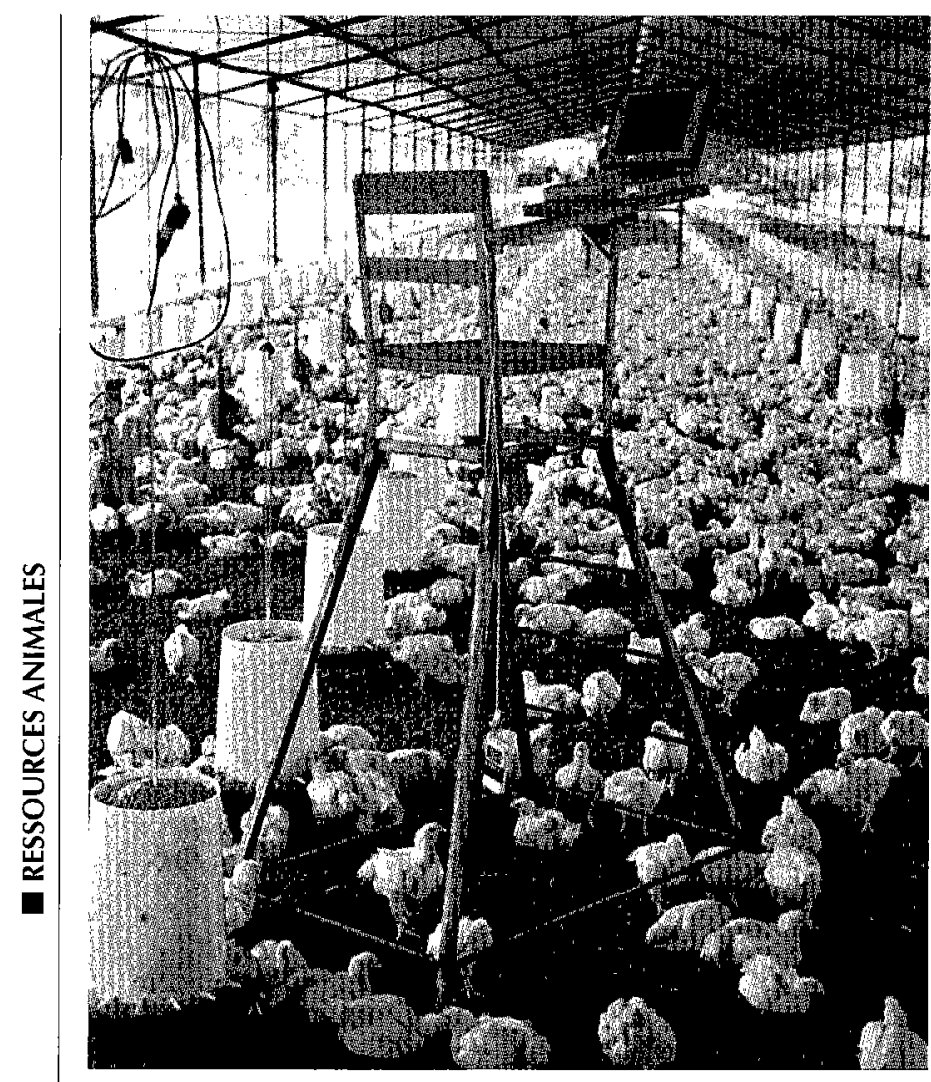

Figure 2 : photo du poste d'observation.

Les enregistrements obtenus par focal sampling permettaient de déterminer la latence, la fréquence, la durée, ainsi que les enchâ̂nements des différentes séquences d'activité du poulet de chair. Ces informations pouvaient être regroupées, pour chacune des activités, sur l'ensemble de la période d'observation (cinq phases), phase par phase, jour par jour ou bien encore par tranche horaire. C'est à partir de ces données totales que les pourcentages de temps consacré à chaque activité (durée totale d'une activité/durée totale des fichiers), la durée des séquences (durée totale d'une activité pour l'ensemble des fichiers/fréquence totale de cette activité), la durée des coups de bec à l'aliment (durée totale de l'activité alimentaire/nombre total de coups de bec correspondants), etc., étaient calculés.

Pour les deux méthodes, les données obtenues étaient analysées (moyennes, erreurs standards, tests non paramétriques, graphiques) à l'aide du logiciel d'analyse statistique Statview.

\section{RESULTATS}

Après avoir éliminé les fichiers de focal sampling d'une durée inférieure à $5 \mathrm{~s}$ (poulet qui se levait lors d'une bousculade et qui se recouchait aussitôt) et ceux de la première phase (trop variables, perte de vue des poussins en cours d'observation, équipements supplémentaires par rapport aux autres phases), les observations par focal sampling ont permis d'obtenir 1716 fichiers (séquences « couché-couché »), soit $29 \mathrm{~h}$ d'observation au total pour quatre phases de quatre jours. Les scannings correspondaient à $16 \mathrm{~h}$ d'observation.
Les résultats des quatre phases ont été présentés en analysant, dans un premier temps, les observations faites par scanning, puis les informations apportées par focal sampling. Seules les principales observations ont été illustrées par des figures. D'autres résultats ont été présentés précédemment et les principales données de base ont été documentées par Richard (14). Les résultats ont été analysés successivement autour de quatre thèmes : occupation de l'espace, évolution des activités en fonction de l'âge, évolution des activités au cours du nycthémère, détection précoce d'un problème d'élevage.

\section{Occupation de l'espace en fonction de l'âge}

$\Lambda$ partir des mesures de scanning, le nombre moyen d'animaux observés dans les différents secteurs du poulailler (mangeoires, abreuvoirs, zones libres) a été divisé par la surface disponible correspondante. La figure 3 représente l'évolution, au cours de la période d'élevage, de la densité des poulets dans les zones libres d'équipement et dans les zones dites «proches » (c'est-à-dire à moins d'un poulet de distance pour ajuster la zone à la taille des poulets) des mangeoires et des abreuvoirs, en distinguant le nombre total de poulets et le nombre de poulets « inactifs » (c'està-dire ne consommant pas ou couchés dans les zones libres).

\section{Nombre "TOTAL" de poulets / m2}

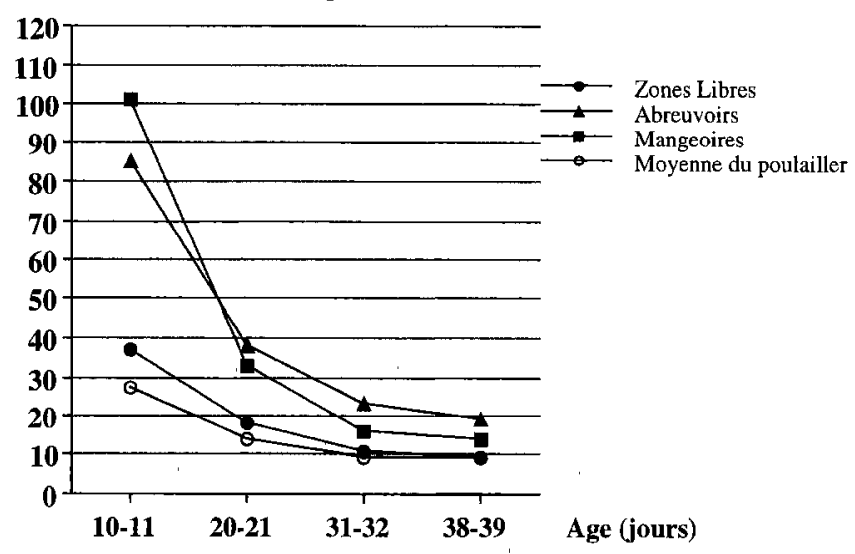

Nombre de poulets "INACTIFS" / m2

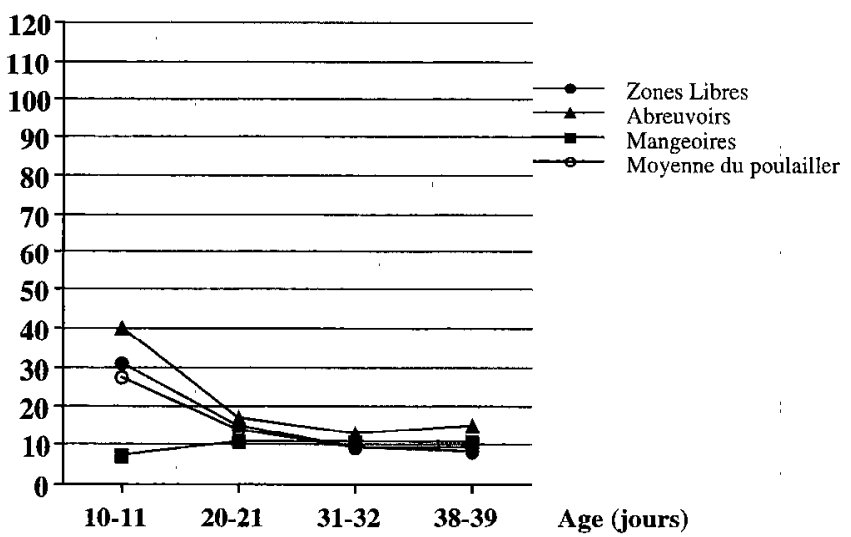

Figure 3 ; - En haut : évolution de la densité totale observée par scanning dans les zones libres, proches des abreuvoirs et mangeoires en fonction de l'âge (poulets $/ \mathrm{m}^{2}, 1$ point = moyenne de 120 mesures) comparée à la densité moyenne de l'élevage.

- En bas : évolution de la densité d'animaux inactifs (couchés ou ne consommant pas) observée par scanning dans les différents secteurs de l'élevage. 
La densité observée au niveau des mangeoires et des abreuvoirs était 2 à 3 fois supérieure à celle mesurée dans les zones libres d'équipement et diminuait au cours de la période d'élevage, proportionnellement à la densité moyenne du poulailler. Cette diminution était cependant plus rapide pour les mangeoires et les abreuvoirs entre les phases 2 et 3 que pour la densité moyenne. Cela était dû à la réduction, avec l'âge, du temps passé dans les activités respectives et aussi à la différence de taille des poulets (et donc de leur encombrement). La croissance des poulets de chair était telle que 100 poulets de 10 jours représentaient une charge animale de $25 \mathrm{~kg} / \mathrm{m}^{2}$ alors que 20 poulets de 38 jours correspondaient à $36 \mathrm{~kg} / \mathrm{m}^{2}$. Le poids rendait cependant mal compte de l'encombrement et de la production de chaleur exacte.

Toutes les mesures relevées par scanning étaient supérieures à la moyenne du poulailler ce qui confirmait un relatif regroupement des animaux vers le centre (partie où les mesures étaient faites, figure 1). De plus, le nombre de poulets « inactifs » près des mangeoires tendait à augmenter avec l'âge.

\section{Evolution des activités du poulet en fonction de l'âge}

L'évolution des activités et des transitions observées par focal sampling est résumée par les schémas de la figure 4 pour les comportements « mange », « boit », « toilette » et « explore ». L'attitude « debout » a été supprimée car il s'agit d'une position intermédiaire entre tous les comportements qui masque les transitions essentielles. La taille des cercles est proportionnelle au pourcentage du temps consacré à chacune des activités considérées, à partir des données de focal sampling; la taille de flèches est proportionnelle au nombre de transitions observées pendant une heure d'activité.

Après s'être levé, le poulet de chair avait tendance, en vieillissant, à privilégier les activités « mange », «boit », et « toilette » au détriment de l'activité « explore ». Par ailleurs, le nombre de transitions entre ces quatre activités lors d'une séquence « couché-couché » diminuait avec l'âge et certaines transitions disparaissaient. Avec l'âge, les séquences d'activité devenaient plus courtes et monocomportementales, le poulet se levait pour réaliser, la plupart du temps, une seule des quatre activités représentées (à l'âge de 40 jours, 417 fichiers sur 458 contenant au moins l'une des activités boire, manger, explorer ou faire une toilette étaient « mono-comportementaux », c'est-à-dire présentaient une seule de ces activités).

Il existe très peu de séquences montrant une activité alimentaire et de consommation d'eau dans le même fichier : les fichiers incluant une séquence alimentaire et une séquence de boisson par rapport aux nombres de fichiers totaux ayant une séquence alimentaire sont au nombre de 17 sur 73 (soit 23 p. 100) à 10 jours, de 9 sur $116(8$ p. 100) à 20 jours, de 0 sur 62 à 30 jours et de 0 sur 176 à 40 jours. Il y avail donc des séquences de consommation d'aliment distinctes de celles de consommation d'eau, ces dernières augmentant avec l'âge sous les effets du climat tropical.

Si seuls les fichiers où une activité finalisée s'était produite (mànger, boire, explorer, faire une toilette) élaient conservés, ce qui revenait à supprimer les fichiers qui ne comportaient que des déplacements, on s'apercevait que le temps d'activité d'un poulet consacré à l'alimentation diminuait avec l'âge (40,8 p. 100 à 9-12 jours, 30,1 p. 100 à $30-32$ jours), mais augmentait (37,5 p. 100) après la mise en service des ventilateurs (37-40 jours). En revanche, le temps consacré à la consommation d'eau augmentait de 9,2 p. 100 à 10 jours à 24,8 p. 100 à 30 jours puis se stabilisait (25,6 p. 100 à 40 jours). Les activités d'exploration relativement importantes (10,6 p. 100 du temps) à l'âge de 10 jours disparaissaient progressivement ( 1,6 p. 100 à 40 jours). Le temps consacré à la toilette demeurait relativement constant $(5,9$ à 8,2 p. $100 \mathrm{du}$ temps). La toilette était un comportement intermédiaire qui précédait plus qu'il ne suivait les activités alimentaires de boisson ou d'exploration.

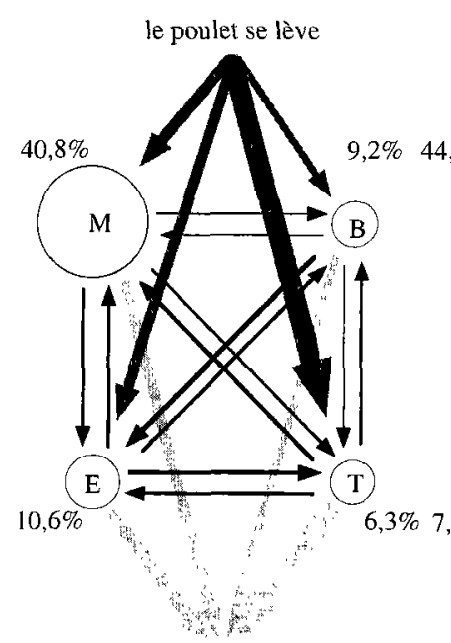

le poulet se recouche Phase $2: 9-12$ jours 211 séquences "couché-couché" de $133,5 \pm 9,5$ secondes

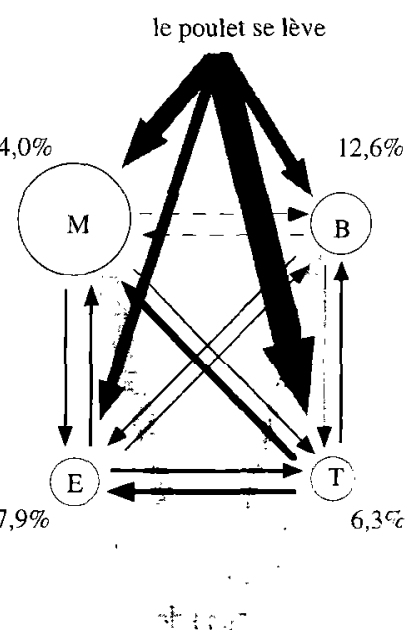

le poulet se recouche

Phase 3 : 19-22 jours 324 séquences "couché-couché" de $88,7 \pm 5,2$ secondes

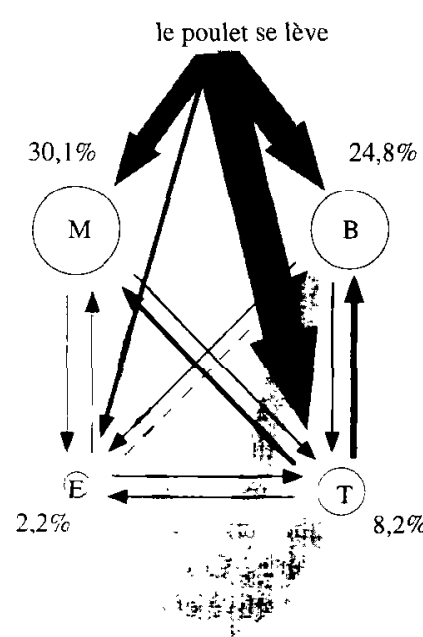

le poulet se recouche Phase $4: 30-32$ jours

246 séquences "couché-couché" de $62,3 \pm 3,2$ secondes

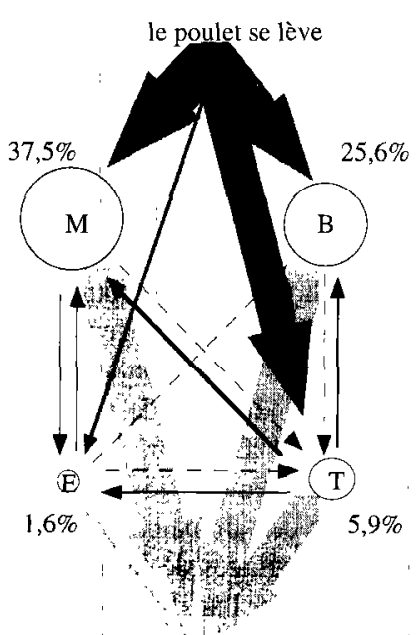

le poulet se recouche Phase $5: 37-40$ jours 458 séquences "couché-couché" de $50,0 \pm 1,6$ secondes

Figure 4 : évolution des activités (cercles de surfaces proportionnelles au pourcentage de temps passé dans chaque activité) et des transitions entre les comportements (flèches d'épaisseurs proportionnelles aux nombres de transitions observées par heure) en fonction de l'âge el d'après les observalions de focal sampling pour toute séquence incluant au moins une des activités (boire, manger, explorer la litière, faire une toilette).

La durée moyenne d'un fichier est suivie de l'erreur standard. 
Si seules les séquences d'activité avec au moins un accès à la mangeoire étaient conservés, la durée moyenne du temps d'alimentation par séquence décroissait de $155 \mathrm{~s}$ (10-12 jours d'âge) à $48 \mathrm{~s}$ cn phase 5 (37-40 jours d'âge) sur une durée moyenne d'une séquence « couché-couché » avec alimentation de 59 s à cet âge (figure 5). La latence, c'est-à-dire la durée enregistrée entre le levé du poulet de chair et son premier accès à la mangeoire, décroissait de $34 \mathrm{~s}$ en phase 2 (10-12 jours) à $7 \mathrm{~s}$ en phase 5 (37-40 jours). Par conséquent, en phase 5 (37-40 jours), le poulet allait directement manger puis se recouchait.

Le comptage des coups de bec pendant une période d'alimentation a permis d'évaluer le rythme moyen de picorage. La durée moyenne de l'intervalle entre deux coups de bec donnés à l'aliment augmentait significativement de $0,73 \pm 0,03 \mathrm{~s}$ en phase 2 (10-12 jours) à $1,15 \pm 0,10 \mathrm{~s}$ cn phase 4 (à $30-32$ jours) et diminuait significativement à $0,88 \pm 0,04 \mathrm{~s}$ en phase 5 (à $37-40$ jours). Ainsi le rythme de picorage était plus rapide en phase 5 qu'en phase 4 , période correspondant à la mise en marche des ventilateurs.

\section{Variation de l'activité des poulets de chair au cours du nycthémère}

Lorsque les fichiers de focal sampling étaient classés par tranche horaire (4-6 h, 7-9 h, 10-12 h, 13-15 h, 16-18 h et 20-22 h), la répartition du temps moyen passé dans chaque activité variait assez peu au cours du nycthémère (en moyenne, 37 p. 100 « debout », 35 p. 100 «mange », 16 p. 100 « boit », 6 p. 100 « toilette » et 6 p. $100 \ll$ explore la litière $»)$. Par contre, la durée moyenne d'une séquence d'activité « couché-couché » variait au cours d'une journée. Tous âges confondus, les séquences étaient plus longues (65-80 s) en début et en fin de journée (éclairage artificiel, températures plus basses) et étaient plus courtes (55 s) en unilieu de journée (lumière et chaleur tropicales). La décomposition par phase des courbes de la figure 6 ne remet pas en cause l'observation faite du poulet qui règle son activité au cours de la journée en faisant varier la durée d'une séquence d'activité plutôt que la composition des différentes activités exprimées.

\section{Détection précoce d'un problème d'élevage}

Les observations comportementales peuvent également permettre de détecter de manière précoce certains problèmes d'élevage. Un second élevage (B) a été observé selon un protocolc similaire à celui décrit ici. Dans ce nouvel élevage, un retard de croissance de 221 g par poulet par rapport au premier élevage (A - déjà décrit) et aux standards vénézuéliens a été mesuré à l'âge de 28 jours et a nécessité un traitement vétérinaire (14). Les deux élevages recevaient des aliments de même origine et de valeur nutritionnelle comparable. Bien que non identifié avec certitude, ce problème d'élevage semble lié à un renouvellement des équipes de surveillance des animaux ayant entraîné quelques erreurs, comme de mauvais réglages de la hauteur des mangeoires. Comme souvent sur le terrain, l'analyse des causes est rétrospective et donc hypothétique. Elle se produit lorsque la baisse de production est mesurée au moment des contrôles de poids systématiques. L'analyse des mesures comportementales a suivi cette même logique car l'observateur ignorait, au moment où les mesures étaient prises, le sens des variations enregistrées.

En comparant les pourcentages d'activité obtenus par focal sampling, on observe, dès l'âge de 20 jours, une différence significative (Mann et Whitney, $\mathrm{P}<0,01$ ) des pourcentages de temps passé à manger ou à explorer : l'activité « mange » représentait 42,9 p. 100 de l'activité des poulcts de l'élevage $\Lambda$, elle ne représentait que 23,2 p. 100 de l'activité des poulets de l'élevage B ; cet écart se réduisait à 30 jours après traitement des animaux $(26,7 \mathrm{p} .100$ pour l'élevage A, 21,1 p. 100 pour l'élevage B).
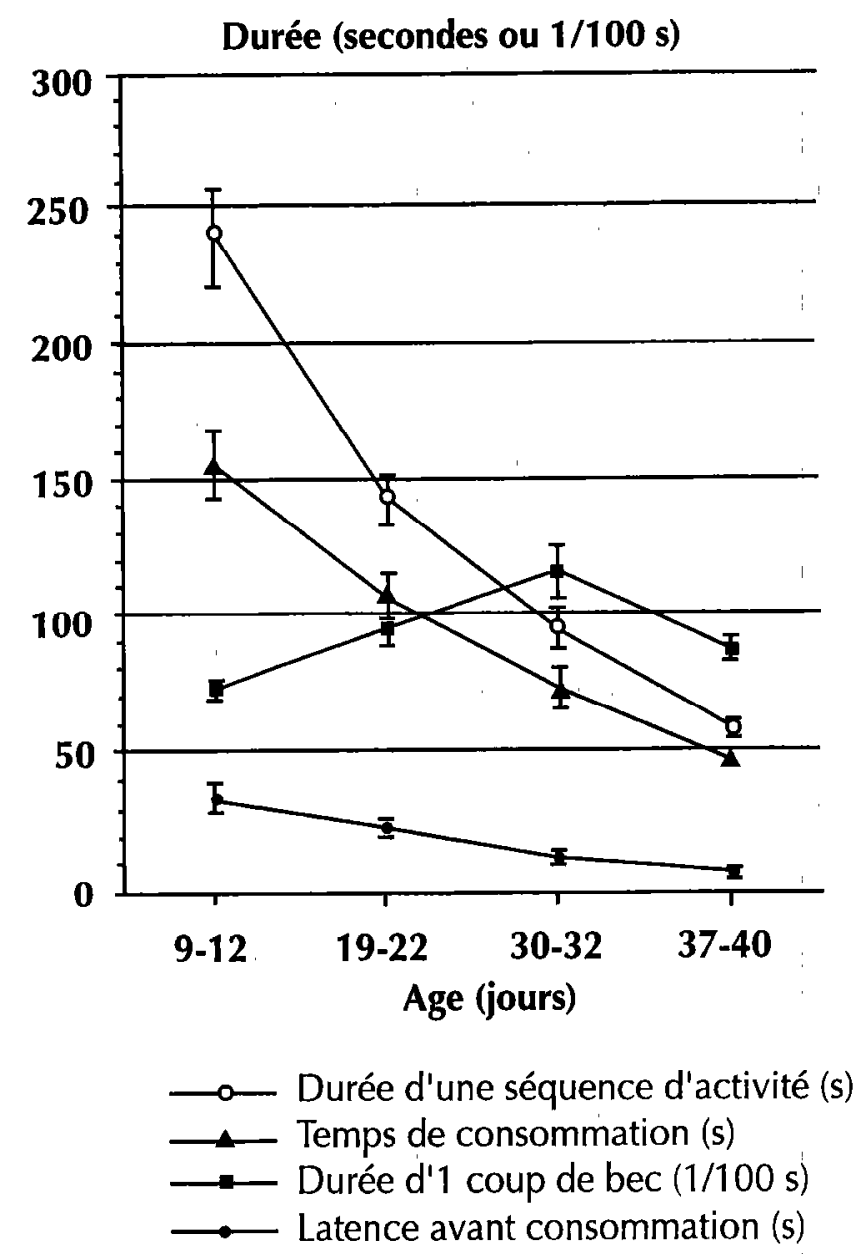

Figure 5 : variation du comportement alimentaire en fonction de l'âge. Moyenne et erreur standard des séquences "couchćcouché "de focal sampling incluant une activité alimentaire, soit respectivement $73,116,62,176$ fichiers pour chaque âge.

Une importante augmentation du temps d'exploration de la litière a été observée simultanément dans l'élevage B : à 20 jours, les poulets de l'élevage A occupaient 4,4 p. 100 de leur temps à explorer, contre 19,5 p. 100 pour les poulets de l'élevage B. A 30 jours, l'écart se réduisait ( 2,3 p. 100 en A, contre 1,3 p. 100 en B). Dans une moindre mesure, des différences entre les pourcentages des mêmes activités dès l'âge de 10 jours $(41,8$ p. 100 en A contre 31,2 p. 100 en $B$ pour le temps passé à manger, et 10,1 p. 100 contre 17,3 p. 100 pour le temps passé à explorer) pouvaient être observées. D'autres mesures comme le temps passé « debout » ou la distance moyenne des animaux aux mangeoires, différaient entre les deux élcvages en phases 2 et 3 . $\Lambda$ insi, il semble qu'une différence de comportement entre les poulets des élevages $\mathrm{A}$ et $\mathrm{B}$ ait été décelable bien avant qu'un retard de croissance n'ait pu être constaté.

\section{DISCUSSION}

Les résultats résumés ici illustrent la richesse des informations issues d'une observation précise des poulets dans les conditions de la production. Il faut cependant souligner que ce travail initial n'a été réalisé que dans un seul élevage où la croissance n'était pas perturbée. La généralisation serait dangereuse et il semble préférable d'émettre des hypothèses pour poursuivre ce type d'étude. 

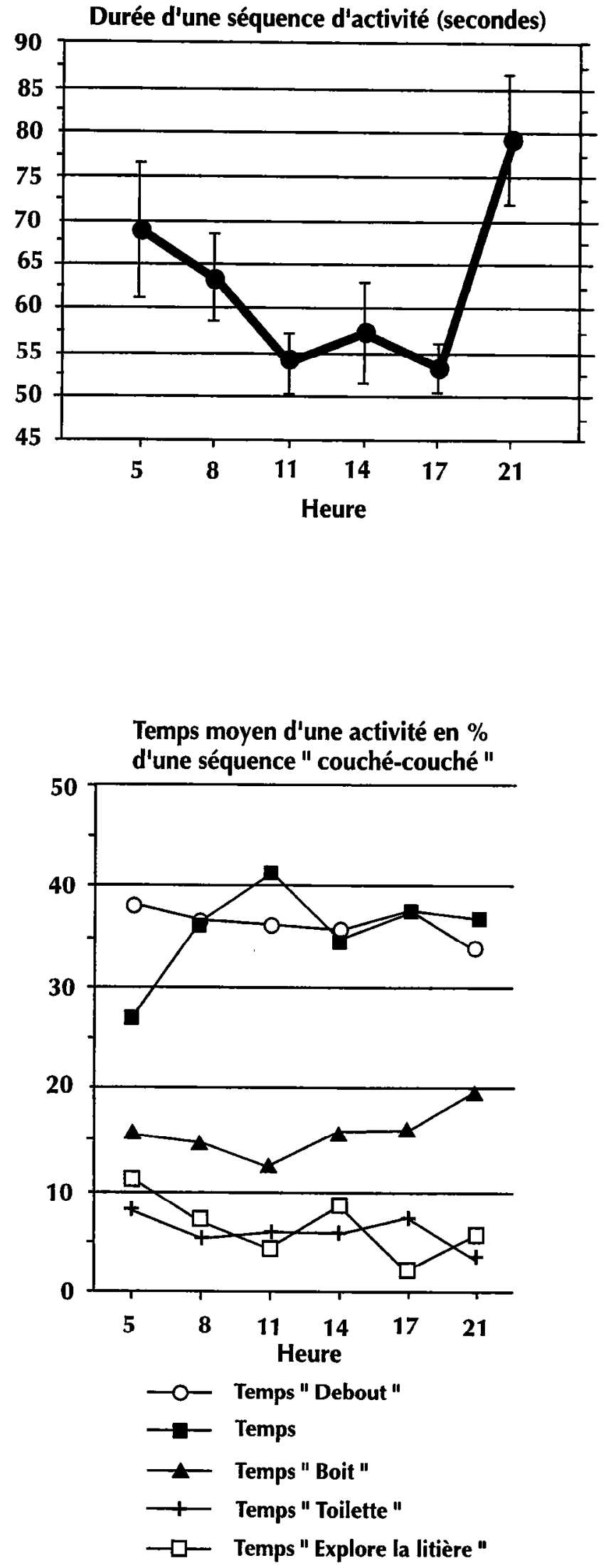

Figure 6 : variation des activités (en bas) et de la durée moycnnc d'un fichier (en haut) en fonction du nycthémère. Chaque point représente la moyenne des focal samplings (respectivement 158,225, 432, 232, 461 et 208 séquences " couché-couché "/tranche horaire).
La réduction d'activité observée chez le poulet de chair avec l'âge est une évolution bien connue (1). En milieu tropical, un poulet de chair couché dans une litière en fermentation qui réduit ses possibilités de pertes thermiques latentes est obligé d'accroitre la thermolyse par évaporation pulmonaire. Les taux de mortalité sont donc plus élevés en finition lors des périodes de chaleur mais ils peuvent être limités par la restriction d'accès à l'aliment pour limiter la production de chaleur métabolique pendant les périodes diurnes (10). Il est aussi de pratique courante au Venezuela de payer des employés pour obliger les poulets en finition à se déplacer pendant les heures les plus chaudes de la journée pour favoriser les échanges thermiques. De même, le brassage d'air par ventilateur a deux effets : il favorise les échanges thermiques en renouvelant l'air au niveau des animaux et il stimule aussi les mouvements des poulets qui n'aiment pas les courants d'air et recherchent donc une position abritée.

La réduction importante (de 133,5 à 50 s entre 10 et 38 jours) des durées moyennes de séquences d'activité incluant un comportement finalisé (manger, boire, faire sa toilette, explorer la litière) suggèrent que le poulet ajuste essentiellement son activité en restant moins longtemps debout lorsqu'il s'est levé. La durée d'une séquence d'activité semble aussi pour le poulct une manière de s'ajuster aux variations nycthémérales d'environnement climatique (température, humidité, lumière). L'es volailles présentent, en général, un pic de consommation d'aliment en début et/ou en fín de photopériode suggérant une certaine capacité de compensation ou d'anticipation sur la période nocturne d'indisponibilité d'aliments (16). En milieu tropical, chez la poule pondeuse, « l'anticipation », ou plus simplement l'adaptation, est limitée aux variations d'intensité lumineuse, l'éclairage naturel diurne étant beaucoup plus intense que l'éclairage artificiel nocturne. Les variations de durée des séquences pourraient être aussi une forme d'adaptation aux variations thermiques de l'environnement. Les volailles n'ont pas de stratégie alimentaire figée, leur activité alimentaire est souvent le résultat d'une adaptation à un environnement (17). Ainsi, la présente étude confirme cette adaptation du poulet de chair à son environnement puisqu'il fait varier, au cours de la journée, la durée de ses périodes d'activité (séquence " couché-couché ») mais il conserve globalement la même répartition des différentes activités (boire, manger, faire une toilette, explorer) au cours d'une période d'activité « couché-couché ». Avec l'âge (et avec l'accentuation des effets de la chaleur sur son métabolisme ?), au contraire, le poulet réduit au minimum les comportements d'exploration $(10,6$ p. 100 du temps à 10 jours contre 1,6 p. 100 à 38 jours) et augmente le temps consacré à l'abreuvement. Tout se passe comme si le poulet en finition optimisait ses activités pour préserver un « repos » d'une durée la plus longue possible.

L'animal peut s'adapter à son environnement de trois manières non exclusives : il peut augmenter ou diminuer le rythme de ses périodes d'activité, augmenter ou diminuer la durée de ces périodes, ou enfin faire varier le contenu de ces périodes. L'évolution comportementale des séquences d'activité avec l'âge vers une seule activité, ce qui limite les transitions, est-elle la conséquence de la rćduction de la durée d'une séquence ou bien l'expression d'un comportement plus finalisé ? La réduction du temps d'exploration, le séjour des poulets plus près des'mangeoires et abreuvoirs suggèrent que les deux évolutions sont probablement concomitantes dans de « bonnes » conditions d'élevage. Picard et coll. (9) ont remarqué que des poulets de 15 jours d'âge alimentés avec un régime déséquilibré en acides aminés explorent plus la litière que des poulets de même âge recevant une alimentation équilibrée ( 4,8 p. 100 contre 1,3 p. 100$)$. Les problèmes techniques dans le second élevage $(\mathrm{B})$ induisent de manière similaire une augmentation importante du comportement d'exploration de la litière. 
L'intérêt pratique évident d'une mesure de la durée des séquences d'activité et de certains comportements comme l'exploration de la litière en fonction de plusieurs paramètres d'élevage serait accru par une meilleure évaluation des phases de « repos » ou par un décompte précis de la fréquence des séquences d'activité, mais cette dernière mesure est difficile dans un élevage de plusieurs milliers d'animaux. En effet, la manière dont le scanning et le focal sampling sont pratiqués ici ne permet pas une évaluation précise de la durée totale du « repos » ni de ce que fait le poulet pendant ce repos. Le focal sampling pourrait être appliqué à des périodes de repos pour décomposer les périodes de sommeil, de polypnée thermique, d'interaction sociale, de toilette. Le scanning devrait, s'il était essentiellement orienté sur l'évaluation du temps de « repos », être conduit de manière plus précise.

Des remarques pratiques proposées par Richard (14) suggèrent qu'une observation par scanning pendant une heure est inutile, voire moins exacte, qu'une observation plus courte. Au cours d'une heure, des perturbations extérieures ou liées aux pratiques périodiques en élevage dérangent les animaux et modifient de manière irrégulière les relevés. En période calme, dix minutes donnent des résultats très semblables à une heure, alors qu'en période de perturbation les relevés d'une heure entière représentent probablement mal l'activité moyenne des animaux au cours de $24 \mathrm{~h}$. Le choix des zones d'observation est, de la même manière, sujet à une analyse critique. Les résultats présentés montrent que la répartition des poulets dans le poulailler n'est pas homogène. L'importance pratique de cette question pour optimiser l'équilibre thermique du troupeau et l'organisation de l'espace et des équipements, notamment en finition, justifie des études complémentaires. Il sera nécessaire de vérifier, par exemple, la moindre utilisation par les poulets au cours du nycthémère des zones du poulailler éclairées directement par le soleil (pendant quelques heures de la journée).

Dans un poulailler, l'ensemble des paramètres environnementaux interviennent et il serait probablement dangereux de trop focaliser les observations sur le seul comportement alimentaire. En considérant l'ensemble des fichiers (comportant une séquence alimentaire ou non), on constate que le pourcentage de temps passé à manger diminue entre les phases 2 à 4 de 41,8 à 26,7 p. 100, mais augmente entre les phases 4 et 5 , après la mise en service des ventilateurs, de 26,7 à 36,6 p. 100. Ce résultat est peut être associé à l'effet de la ventilation qui semble faire augmenter le nombre de séquences avec consommation alimentaire par rapport à l'activité totale du poulet et le rythme de picorage sans modifier la durée moyenne des séquences alimentaires. Entre les phases 4 et 5 (soit 30 et 40 jours d'âge), période de mise en marche des ventilateurs, la durée d'un coup de bec donné à l'aliment a diminué de 1,15 à 0,88 s. Savory (15) a montré qu'une température d'élevage élevée a un effet dépressif sur le rythme d'ingestion de l'aliment (c'est-àdire la quantité d'aliment ingérée par unité de temps d'accès alimentaire). Le rythme de picorage peut être associé à une variation de perception de la chaleur ou à l'effet des mouvements d'air sur l'exécution d'un comportement (2).

$\mathrm{Au}$ cours d'un « séjour » à la mangeoire, l'animal donne une série de coups de bec plus ou moins rapides à l'aliment. Certains coups de bec aboutissent à la préhension et à l'ingestion d'aliment (coups de bec consommateurs) et d'autres coups de bec sont exploratoires (20). Vilariño et coll. (18) ont observé que le rythme de picorage (nombre de coups de bec par seconde d'accès alimentaire) des poules pondeuses est plus lent avec un aliment granulé. Un changement d'aliment (remplacement de l'aliment farine par de l'aliment granulé) a eu lieu au $20^{\mathrm{e}}$ jour d'élevage, c'est-à-dire en phase 3. La même augmentation de la durée d'un coup de bec a été observée entre les phases 2 et 3 d'une part et les phases 3 et 4 d'autre part, suggérant que cette augmentation est plus liée à l'âge du poulet ou à l'effet de la température qu'à la granulation de l'aliment. Le fait que l'aliment granulé était très friable renforce cette hypothèse.

Murphy et Preston (7) ont observé que 48 p. 100 des accès aux mangeoires durent moins de $60 \mathrm{~s}$ pour des poulets de chair de 27 à 50 jours d'âge recevant un aliment complet en granulés et élevés en conditions intensives pratiques (durée moyenne de ces accès : $74 \mathrm{~s}$ ). Picard et coll. (11), sur la base d'un critère de « fin d'accès alimentaire » fixé à $5 \mathrm{~s}$ d'interruption, ont mesuré des durées moyennes de respectivement 60 et $81 \mathrm{~s}$ pour des poulets de 18 jours en cages individuelles avec ou sans contacts visuels, et de $145 \mathrm{~s}$ pour des poulets en groupes de quatre. La fin d'un accès alimentaire correspond généralement au début d'une autre activité. Ce critère de fin est imprécis, notamment lorsque l'animal reste immobile près de la mangeoire. Les résultats obtenus au cours de cette étude sont comparables à ceux des auteurs cités $(7,11)$ puisque les accès alimentaires ont une durée moyenne de 95,5 $\mathrm{s}$ entre les phases 2 à 5 (soit 10 à 40 jours d'âge ; élevage A) et de $60 \mathrm{~s}$ pour les phases 4 et 5 (soit 30 à 40 jours d'âge). La réduction de la durée d'un accès à la mangeoire avec l'âge peut être interprétée comme le résultat d'une plus grande efficacité de la préhension des particules alimentaires et/ou comme une adaptation à des contraintes environnementales (perception de la chaleur et de l'hygrométrie, mouvements d'air, accès limité par le séjour d'autres poulets autour des équipements).

\section{- CONCLUSION}

Les mesures de comportement peuvent être développées en élevage intensif pour mieux évaluer l'occupation de l'espace, les comportements redirigés à la suite d'une erreur ou d'un problème (comme le picorage accru de litière dans l'élevage B) et les fluctuations adaptatives en fonction de la chaleur et de la lumière. Deux directions principales pourraient conduire d'une part vers des mesures de type diagnostic de routine, c'est-à-dire la fréquence du picorage du sol, stéréotypies, et d'autre part vers une approche de l'adaptation en partant de l'hypothèse que le poulet de chair module d'abord la durée d'une séquence d'activité. La conception de modèles combinant la probabilité de l'initiation d'une séquence d'activité, celles de son contenu et de son arrêt sera facilitée par le fait que les poulets de chair tendent à faire des séquences mono-comportementales après quatre semaines d'âge, c'est-à-dire au moment le plus critique de la production sur un plan économique et énergétique.

La mesure précise de la durée des séquences d'activité du poulet de chair dans un élevage peut, sous réserve de validation, devenir une mesure de l'adaptation et de l'effet de plusieurs facteurs environnementaux (climatiques ou techniques). Elle présenterait l'intérêt de ne nécessiter qu'un chronomètre.

L'évaluation des activités d'exploration pourrait révéler certains problèmes d'élevage. Ces activités d'exploration pourraient être mesurées par focal sampling en même temps que la durée des séquences d'activité en comptant le nombre de picorages donnés au sol et aux équipements. Un scanning des attitudes d'exploration est également possible sous réserve d'une définition précise des attitudes. Le terme « exploration» doit être considéré ici comme une facilité d'expression pour désigner des coups de becs non dirigés vers l'aliment, l'eau ou le plumage. 
Le scanning doit être réalisé dans des conditions calmes pendant un temps réduit à environ 10 min mais il semble souhaitable de mieux analyser l'hétérogénéité dans la répartition spatiale des animaux dans le poulailler pour définir la représentativité de la mesure.

Une analyse plus précise des attitudes de repos et surtout du temps réel d'inactivité apparente est un complément indispensable des mesures de séquences d'activité.

Les résultats obtenus au cours de cette étude renforcent l'importance d'établir un lien direct entre la production et la recherche par l'étude du comportement.

\section{Remerciements}

Les auteurs remercient la Direction de Protinal et les propriétaires des élevages de Corazon de Jesus et de Los Cocos qui ont accepté que ce travail se réalise dans leurs unités de production, le FONAIAP et l'INRA pour leur appui financier et le CIRAD-EMVT qui a coordonné le travail de stage du DESS de Productions animales en régions chaudes.

\section{BIBLIO GRAPHIE}

1. BESSEI W., 1992. Das Verhalten von Broilern unter Intensivenhaltungsbedingungen. Arch. G eflügelk., 56: 1-7.

2. FAURE J.M., LAGADIC H., 1994. Elasticity of demand for food and sand in laying hens subjected to variable wind speed. Appl. Anim. Behav. Sci., 42: 49-59.

3. HILL W., ROVEE-CO LLIER C., CO LLIER G., WASSERLO OS L., 1986. Time budgets in growing chicks. Physiol. Behav., 37: 353-360.

4. JOHNSON D.F., COLLIER G., 1991. The relationships between feeding rate and patch choice. J. exp. anal. Behav., 55: 75-95.

5. MARTIN P., BATESON P., 1986. Measuring behaviour. Cambridge, U nited Kingdom, Cambridge U niversity Press, p. 48-69.

6. MAULDIN J.M., 1992. Applications of behaviour to poultry management. Poult. Sci., 71: 634-642.

7. MURPHY L., PRESTON A., 1988. Food availability and the feeding and drinking behaviour of broilers chickens grown commercially. $\mathrm{Br}$. Poult. Sci., 29: 273-283.
8. MURPHY L., PRESTON A., 1988. Time-budgeting in meat chickens grown commercially. Br. Poult. Sci., 29: 571-580.

9. PICARD M., FAURE J.M., SIEGEL P.B., DUNNINGTON E.A., UZU G., DALIBARD P., 1994. Food intake and aminoacids in poultry. Antony, France, Rhône Poulenc Scientific Paper, Rhône Poulenc Animal Nutrition, $44 \mathrm{p}$.

10. PICARD M., SAUVEUR B., FENARDII F., ANGULO I., MONGIN P., 1993. Adjustements technico-économiques possibles de l'alimentation des volailles dans les pays chauds. IN RA Prod. Anim., 6: 87-103.

11. PICARD M., TURRO I., LAUNAY F., MILLS A., MELIN J.M. FAURE J.M., 1992. Food intake patterns of three week old broilers caged individually or in groups. In: 19th World poultry science Congress, Amsterdam, the N etherlands, September 20-24, 1992.

12. PRESTON A.P., MURPHY L.B., 1988. O bservations on the use of feeding space in commercial broiler chicken house. Br. Poult. Sci., 29 293-300.

13. PRESTO N A.P., MURPHY L.B., 1989. Movement of broiler chickens reared in commercial conditions. Br. Poult. Sci., 30: 519-532.

14. RICHARD P., 1996. Etude du comportement du poulet de chair en élevage intensif tropical au Venezuela. Mémoire de stage DESS Productions animales en régions chaudes, CIRAD-EMVT, Montpellier, France, $69 \mathrm{p}$

15. SAVORY C.J., 1986. Influence of ambiant temperature on feeding activity parameters and digestive function in domestic fowls. Physiol. Behav., 38: 353-357.

16. SQ UIBB R.L., COLLIER G.H., 1979. Feeding behaviour of chicks under three lighting regimens. Br. Poult. Sci., 58: 641-645.

17. TURRO -VINCENT I., 1994. O ntogenèse du comportement alimentaire du poussin (Gallus domesticus) dans les conditions de l'élevage intensif. Thèse doct., U niversité François Rabelais, Tours, France, $205 \mathrm{p}$.

18. VILARINO M., PICARD M., MELCION J.P., FAURE J.M., 1996. Behavioural adaptation of laying hens to dilution of diets under mash and pellet form. Br. Poult. Sci., 37 : 895-908.

19. YO T., 1994. Adaptation comportementale au choix alimentaire du poulet de chair et de la poule pondeuse (Gallus domesticus) en milieu tropical. Thèse doct. Sciences biologiques et agronomiques, ENSA, Rennes, France, $267 \mathrm{p}$.

20. YO T., VILARINO M., FAURE J.M., PICARD M., 1997. Feed pecking in young chickens: new techniques of estimation. Physiol. Behav., 61 (6): $803-810$

Reçu le 9.4.97, accepté le 1.7.97 


\section{Summary}

Richard P., Vilariño M., Faure J.M., León A., Picard M. Study of broiler chickens' behaviour in a tropical intensive breeding poultry house in Venezuela

To identify behavioural indicators of chickens' adaptation to their environment, scanning and focal sampling behavioural measurements were recorded in a flock of 19,500 broilers in a tropical intensive breeding poultry house during one production cycle (40 days). The number of birds observed around feeding devices and troughs was 2-3 times higher than the average density in the industrial poultry house. Duration of an activity bout (between two resting bouts) including at least one pecking at feed, water, litter or one preening) was on average $133.5 \pm 9.5 \mathrm{~s}$ at 10 days of age and regularly decreased to $50.0 \pm 1.6 \mathrm{~s}$ at 40 days. With age activity bouts were almost reduced to only one type of behaviour (mainly eating/drinking), as the frequency of the exploratory floor pecking behaviour decreased. O ver a day, duration of an activity bout decreased during the warmest hours and increased again in the evening. However, the ethogram of an active broiler remained the same throughout the day. A management problem could be identified early by observing increased floor pecking activity. Density measurements of animal distribution, activity bout duration and frequency of litter pecking will provide practical advantages if they are very accurately defined and performed under peaceful conditions.

Key words: Broiler chicken - Aviculture - Behaviour Adaptation - Growth - Poultry housing - Intensive husbandry - Floor husbandry - Tropical climate Venezuela.

\section{Resumen}

Richard P., Vilariño M., Faure J.M., León A., Picard M. Estudio del comportamiento del pollo de carne en un criadero intensivo tropical en Venezuela

Con el fin de identificar los indicadores del comportamiento de adaptación al medio, se llevaron a cabo mediciones des comportamiento por scanning y muestreo focal, en un criadero intensivo tropical de 19500 pollos de carne y durante un ciclo de producción (40 días). La densidad animal alrededor de los comederos y bebederos fue 2 a 3 veces superior a la densidad media del criadero. La duración de una secuencia de actividad (« reposo-reposo ») incluyendo al menos un picoteo del alimenteo, de agua, de la cama o una limpieza fue en promedio de $133,5 \pm 9,5$ s a los 10 días y disminuyó regularmente para alcanzar $50,0 \pm 1,6 \mathrm{~s}$ a los 40 días. Con la edad, las secuencias se orientaron casi todas hacia una actividad única (mono comportamiento, principalmente comer o beber), el comportamiento de exploración por picoteo de la cama disminuyó. Durante el curso del día la duración de una secuencia de actividad disminuyó durante los períodos calientes y aumentó durante la noche, pero el pollo, mientras que estaba activo, presentó el mismo etograma a cualquier hora. En caso de problema de crianza, los comportamientos de exploración de la cama aumentaron nuevamente, lo que podía constituir una señal precoz. Las mediciones de la densidad de distribución de los animales, de la duración de una secuencia de actividad y de la frecuencia de picoteo de la cama mostraron un interés práctico, siempre y cuando estén definidascon precision y realizadas en condiciones tranquilas.

Palabras clave: Pollo de engorde - Avicultura Comportamiento - Adaptación - Crecimiento - Alojamiento para aves - Crianza intensiva - Crianza en el suelo - Clima tropical - Venezuela. 